\title{
Publisher's Note: Curvature-induced crosshatched order in two-dimensional semiflexible polymer networks [Phys. Rev. E 92, 060602(R) (2015)]
}

\author{
Cyril Vrusch and Cornelis Storm \\ (Received 4 January 2016; published 11 January 2016)
}

DOI: 10.1103/PhysRevE.93.019902

This paper was published online on 17 December 2015 with an error in the text on page 2. On page 2, the seventeenth line of the left-hand column should read as "the bending modulus $\kappa$ in [J m]. However, we will consider..." The paper has been corrected as of 21 December 2015. The text is correct in the printed version of the journal. 